\title{
Translating Western Animal Models into TCM
}

\author{
Changqing Pang \\ Department of Basic Courses, Shandong University of Science and Technology, Jinan, Shandong, China.
}

\begin{abstract}
How to cite this paper: Changqing Pang. (2021). Translating Western Animal Models into TCM. The Educational Review, USA, 5(11), 447-450.

DOI: 10.26855/er.2021.11.005
\end{abstract}

Received: October 12, 2021

Accepted: November 6, 2021

Published: November 30, 2021

Corresponding author: Changqing Pang, Department of Basic Courses, Shandong University of Science and Technology, Jinan, Shandong, China.

Email: 13853184438@163.com

\begin{abstract}
To translate western animal models into TCM means to combine TCM aetiological characteristics and pathobiology of western medicine to establish animal models. Translating western animal models into TCM does provide us with a new approach which will be able to help TCM diagnose diseases on more objective basis and quantitative basis. Traditionally, animal models of traditional Chinese medicine (TCM) are not easily reproducible, where syndromes are mostly appreciated. Most duplicated animal models could hardly reflect the natures and whole pictures of diseases. In addition, the modeling method based on disease syndromes could only reflect single aspect of a disease, since different diseases may most probably share the same syndromes. On the opposite side, western models appreciate diseases as the first priority. Anyhow, both separate the relationship between diseases and syndromes. Translating the animal models of western medicine into the experimental research of traditional Chinese medicines has the advantages of reliable results and high degree of recognition. The diseases-and-syndrome-combined animal models are more reliable and stable, allowing consistent and dynamic observation on macro and micro variations happened on such models, so as to very closely simulate clinical experiences.
\end{abstract}

Keywords

Translating, Western Animal Models, TCM

\section{Introduction}

It is the necessary trend to combine clinical diagnosis, treatment and research of diseases and syndromes, which has been reflected in the development of traditional Chinese medicine, where western medicine ideology and methods are encouraged to be translated into its Chinese counterpart. Up to now, the ideology of integration of the two different medical systems has become more and more mature, but we are still having a long way to go toward perfecting TCM effects. Animal experiment is the basis and condition of medical development, which is very typical for western counterpart since it has been contributing greatly to most great medical achievements. Compared with the traditional animal model of traditional Chinese medicine and the animal model of etiology and pathology of western medicine, the combination of disease and syndrome with animal model has obvious advantages in the present generation of traditional Chinese medicine, where the western-Chinese translation work has to be given the first priority.

\section{Traditional principle animal modeling methods of TCM}

Animal experiment is one of the important means of medical research. Experimental animals are compared to "living precision instruments", which are closely related to the development and progress of medicine. At present, animal experiment is also one of the common methods of traditional Chinese medicine research. Experimental animals can replace 
human body to study the toxic and side effects of traditional Chinese medicine, scientifically evaluate the curative effect of traditional Chinese medicine, and provide reference for the development of traditional Chinese medicine theory (Shen, 2020). Traditionally, animal model of traditional Chinese medicine etiology has been taking a firm position, but gradually diminishing its occupation to less than 20 percent of the whole market. Simulating disease causes has been the core of animal modeling for traditional Chinese medicine, which can be divided into two kinds of modeling methods: single factor modelling and compound factors modelling.

\subsection{Single factor modelling}

Single factor modelling stresses on single factor which is presumably regarded as the only or main cause leading to a disease. According to one TCM theory, the strong feeling of fear will hurt kidney, two kinds of animals of cats and mice are selected to establish disease cause models, since it is very obvious that mice will be very scared at the sight of cats. In this way, the animal model of kidney deficiency is established by "cat scaring mice" so as to find how much a human's kidney can be endangered or hurt by the feeling of fear, and then develop specific animals with kidney problems. Similarly, based on the TCM theory "anger injures liver", the animal model of liver depression syndrome is developed by means of irritating stimulation method to find out what animals will easily catch liver diseases and to eventually foster animals with liver problems. According to the theory of "wind-cold-dampness" theory, an animal model of wind-cold-dampness arthralgia was developed by using artificial wind-cold-dampness environment device (Li, 2014).

\subsection{Compound factors modelling}

Compound factors modelling applies more than one factors to force certain animals to catch certain diseases. For example, TCM believes that the deficiency of heart strength is generally caused by over-labor and fear, which is why mice are choose to be frightened and forced to over-run to establish the model of heart deficiency. Another example is about the theory that blood deficiency syndrome will arise if blood does not naturally generate while blood consumes excessively in some ways. Accordingly, the animal model of "blood deficiency" by means of bloodletting and restricted drinking and eating is established (Ma, 2014).

The advantages of the two traditional modeling methods lie in that the selection of animal models is based on etiology of TCM, where origins of disease are greatly taken into account, and results of experiments are more consistent with the traditional Chinese medicine theory because of the agreement between disease origin, symptom, physical data and prescription. Generally, the experimental result agrees with the corresponding TCM theories, which is beneficial to the disclosure of the essential verification of disease syndrome, and then helps a lot in discovering effective TCM treatments, as well as new breakthroughs in developing traditional Chinese medicine theories.

However, the defects of the traditional animal modeling can also be easily seen in the following aspects. First of all, the unique nature of a syndrome of a disease has to be the only factor to be used to duplicate animal models, but factually, natures of most diseases are still ambiguous. In this way, most duplicated animal models could hardly reflect the natures and whole pictures of diseases. Secondly, the modeling method based on disease syndromes could only reflect single aspect of a disease, since different diseases may most probably share the same syndromes. In other words, diseases and syndromes are correlated in very complicated ways, not in the way of simple correspondences. In this way, the models established in this way are not sufficiently representative.

\section{Animal modeling based on etiological and pathological mechanism of western medicine}

Experimental zoology is an interdisciplinary subject, which mainly involves animal science and animal medicine. It is a basic and technical subject for medicine, biology and pharmacy (Zhang, 2017). It is most prevalent nowadays that animal models are made based on pathological damages of animal tissues, organs, even the whole body, by means of specific disease-inducing factors chemically, biologically, mechanically and physically, which is being extensively used.

Firstly, chemical stimulation is generally applied in establishing animal models, for example, dexamethasone is fed to rabbits to set up models where their glucocorticoid is restrained, and then it is found that the Chinese medicine Shengdi is able to alleviate the adverse effect on rabbits' adrenal gland by glucocorticoid. Another example is about the blood stasis model of rabbits simulated in the way where macromolecule polymer dextran solution is injected into the ear margin veins of rabbits, which leads to the discovery of Chuanxiong Injection, an effective cure for blood stasis.

Secondly, biological stimulation is also very popular for animal modeling. The model of the disease Internal injury rheumatism is established by injecting blood serum and tissue fluid into hybridized rats between New Zealand black rats and Kunming white rats, since they carry antinuclear antibodies, which is very similar to human being's. 
Thirdly, modeling approaches can also be found in physical and mechanical stimulations. To simulate local cerebral ischemia, middle arteries of a rat are ligatured. On this basis, acupuncture is proved to be effective to cure local cerebral ischemia after measuring the conditioned reflex, cerebral infarction size and brain tissue pathology (Liu, 2013).

The advantages of establishing such models in this way lie in that the methods are stable, the experiment results are reliable and can be easily reproduced, and they are in good comparability with the results of modern medical research, especially in the aspect of pharmacological research of new Chinese medicine. On the other hand, disadvantages are also obvious where such kinds of modeling methods are based on western medicine theories, and western medicine indicators are used as regulations on TCP, which leads to that TCP stands in the position of being studied and interpreted. Since TCP beliefs in the origins of diseases are different from western medicine, pharmacological study loses its dialectical treatment basis, obviously not in accordance with TCP pharmacology, which will have adverse effect on the study on the natures of disease symptoms.

\section{Translating western animal models into TCM}

To translate western animal models into TCM means to combine TCM etiological characteristics and pathobiology of western medicine to establish animal models. Factually, this approach has not been widely developed. Experimental zoology comes from many disciplines and is applicable to many disciplines, while the development of TCM in this field cannot meet the needs of today's multi-disciplinary, multi-angle and multi-level researchers for Experimental Zoology Knowledge and skills (Zhang, 2017). Anyhow, this approach draws successful experiences of TCM and western medicine, while exhibiting respective strong points. Animal models established based on the combination of the two distinct theories not only associate closely with TCM theories, but also are in close accordance with some diseases nowadays, which is beneficial to the theoretical study on the combination of TCM and western medicine. However, the common things between the two medical systems are still waiting to be identified, and the working effects of the combined research are still waiting to be developed. In this way, it is still not easy to establish models in this approach.

One of the effective example goes like this: in order to establish the model of spleen deficiency in rats, over-eating and over-working are adopted. Furthermore, according to western medicine theories, aluminum chloride is used to simulate the disease of Alzheimer. In this way, two symptoms are reflected in the subject of rats. Then, the prescription of tonifying spleen and stomach is used to test whether the TCM treatment is effective. Luckily, it works very well on the two diseases.

Nevertheless, by translating western disease animal models into TCM, diseases and syndromes are closely related with each other, typically supported by recent clinical practices, and has become the latest trend of Chinese medical experimental animal modeling, which is commonly known as the diseases-and-syndrome-combined animal modeling. TCM deals with diseases by identifying syndromes to prescribe, while western medicine identifies the specific diseases before prescription. They both try to find cures from different perspectives. The diseases-and-syndrome-combined animal models are more reliable and stable, allowing consistent and dynamic observation on macro and micro variations happened on such models, so as to very closely simulate clinical process (Lu, 2021). In addition, such models help a lot in establishing quantitative diagnostic criteria, syndrome efficacy evaluation system and curative effect judgment criteria, as well as the mechanism research on same diseases with different syndromes. Meanwhile, the combined modeling approach signifies the rapid development of new Chinese medicines, expanding TCM widely into the world.

The study on type II diabetes model guided by disease-and-syndrome-combined animal modeling approach will serve as a typical example of the trend. At the very beginning, rats are fed with fatty food. Four weeks later, food fasting is applied in addition to water, which lasts for twelve hours. After that, streptozotocin (35mg/kg) is injected into the rats. Five days later, random glucose level is tested. If random glucose level is higher than $16.7 \mathrm{mmol} / \mathrm{L}$, type II diabetes model are established. And all rats with type II diabetes are classified into five groups, namely diabetes group, Yin-yang deficiency group, blood-stasis-and energy-stagnation group, yin-deficiency-and-heat sufficiency type group, and energy-deficiency-and-yin-deficiency type group. Then different medicines are given to four groups except group one. Yin-yang deficiency group is served with olacaceae, fructus aurantii, dried ginger, and cypress; blood-stasis-and energy-stagnation group is served with rhizoma coptidis and gypsum; yin-deficiency-and-heat sufficiency type group is served with dried ginger, aconite, cinnamon, and ligustrum lucidum; energy-deficiency-and-yin-deficiency type group is served with Olacaceae, fructus aurantii, and aconite. Another four weeks later, half of the rats are killed to be tested, and the rest rats are served with the same medicines. And then, the rest rats are killed after the new four weeks. The results indicate that the different syndrome types exhibit variations and objective changes, which proves that it is practical to establish more pragmatic type II diabetes models in the syndrome-and disease-combined modeling approach (Wu, 2013).

Factually, TCM properties are not characterized typically in evaluating animal models. In TCM, four ways of diag- 
nosis of looking, listening, questioning and pulse feeling lay the foundation for detecting disease syndromes, which is hard to do on animals. The question lies in how to summarize information collected from animal models to classify them into different syndromes. Translating western animal models into TCM does provide us a new approach which will be able to help TCM diagnose diseases on more objective basis and quantitative basis.

\section{References}

Aihua Zhang, Wentao Zeng. (2017). Practice and exploration on teaching reform of laboratory animal science in medical universities. Chinese Journal of Comparative Medicine, 3, 94.

$\mathrm{An} \mathrm{Wu}$, et al. (2013). Correlation of syndrome and variance of laboratory targets of type II diabetic disease syndrome combination rat models. Chinese Archives of Traditional Chinese Medicine, 9, 37-41.

Xiaogou Lu, et al. (2021). Review on animal experiment and clinical research of traditional Chinese medicine emotional therapy. China Journal of Traditional Chinese Medicine and Pharmacy, 1, 347.

Xiaojuan Li, et al. (2014). Development and prospect of establishment method of traditional Chinese medicine animal models. China Journal of Traditional Chinese Medicine and Pharmacy, 7, 76-78.

Xueling Ma, et al. (2013). On TCM Syndrome and Biological Mechanism of Spontaneously Hypertensive Rats. World Chinese Medicine, 2, 46-48.

Yang Shen, et al. (2020). Effect of Species Difference of Experimental Animals on Traditional Chinese Medicine Research. Chinese Medicine Modern Distance Education of China, 9, 152.

Yueyun Liu, et al. (2013). Behavior evaluation of rat model with stagnation of liver qi and spleen deficiency syndrome regulating by Xiaoyao San extract. Chinese Archives of Traditional Chinese Medicine, 7, 92-95. 